\title{
Use of multiparametric MRI to characterize uterine fibroid tissue types
}

\author{
Inez M. Verpalen ${ }^{1} \cdot$ Kimberley J. Anneveldt ${ }^{1,2} \cdot$ Pieter C. Vos ${ }^{4} \cdot$ Mireille A. Edens ${ }^{3} \cdot$ Edwin Heijman $^{4,5}$. \\ Ingrid M. Nijholt ${ }^{1}$. Jeroen R. Dijkstra ${ }^{2}$. Joke M. Schutte ${ }^{2}$. Arie Franx ${ }^{6} \cdot$ Lambertus W. Bartels $^{7}$. Chrit T. W. Moonen ${ }^{7}$. \\ Martijn F. Boomsma ${ }^{1}$
}

Received: 29 November 2019 / Revised: 2 March 2020 / Accepted: 10 March 2020

(c) European Society for Magnetic Resonance in Medicine and Biology (ESMRMB) 2020

\begin{abstract}
Background Although the biological characteristics of uterine fibroids (UF) have implications for therapy choice and effectiveness, there is limited MRI data about these characteristics. Currently, the Funaki classification and Scaled Signal Intensity (SSI) are used to predict treatment outcome but both screening-tools appear to be suboptimal. Therefore, multiparametric and quantitative MRI was studied to evaluate various biological characteristics of UF.

Methods 87 patients with UF underwent an MRI-examination. Differences between UF tissues and myometrium were investigated using T2-mapping, Apparent Diffusion Coefficient (ADC) maps with different $b$-value combinations, contrastenhanced T1-weighted and T2-weighted imaging. Additionally, the Funaki classification and SSI were calculated.

Results Significant differences between myometrium and UF tissue in T2-mapping $(p=0.001)$, long-TE ADC low $b$-values $(p=0.002)$, ADC all $b$-values $(p<0.001)$ and high $b$-values $(p<0.001)$ were found. Significant differences between Funaki type 3 versus type 1 and 2 were observed in SSI $(p<0.001)$ and T2-values $(p<0.001)$. Significant correlations were found between SSI and T2-mapping $\left(p<0.001 ; \rho_{s}=0.82\right)$, ADC all $b$-values $\left(p=0.004 ; \rho_{s}=0.31\right)$, ADC high $b$-values $(p<0.001$; $\left.\rho_{s}=0.44\right)$ and long-TE ADC low $b$-values $\left(p=0.004 ; \rho_{\mathrm{s}}=0.31\right)$.

Conclusions Quantitative MR-data allowed us to distinguish UF tissue from myometrium and to discriminate different UF tissue types and may, therefore, be a useful tool to predict treatment outcome/determine optimal treatment modality.
\end{abstract}

Keywords Uterine fibroids · High-intensity focused ultrasound ablation · Magnetic resonance imaging · Diffusion MRI

Electronic supplementary material The online version of this article (https://doi.org/10.1007/s10334-020-00841-9) contains supplementary material, which is available to authorized users.

Inez M. Verpalen

i.m.verpalen@isala.nl

1 Department of Radiology, Isala Hospital, Dokter van Heesweg 2, 8025 Zwolle, The Netherlands

2 Department of Gynecology, Isala, Zwolle, The Netherlands

3 Department of Innovation and Science, Isala, Zwolle, The Netherlands

4 Oncology Solutions, Philips Research, Eindhoven, The Netherlands

5 Department of Diagnostic and Interventional Radiology, Faculty of Medicine and University Hospital of Cologne, University of Cologne, Cologne, Germany

6 Department of Obstetrics and Gynaecology, Erasmus Medical Center, Rotterdam, the Netherlands

7 Imaging Division, Image Sciences Institute, University Medical Center Utrecht, Utrecht, The Netherlands

\section{Abbreviations}

ADC Apparent diffusion coefficient

DWI Diffusion-weighted imaging

MR-HIFU Magnetic resonance image guided-high intensity focused ultrasound

MRI Magnetic resonance imaging

NPV Non-perfused volume

SI Signal intensity

SSI Scaled signal intensity

TE Echo time

tSSS Transformed symptom severity score

tHRQL Transformed health-related quality of life

UAE Uterine artery embolization

UFS-QoL Uterine fibroid symptom health-related quality of life questionnaire

UF Uterine fibroids 


\section{Introduction}

Uterine fibroids (UF) are the most common benign smooth muscle cell tumors in the pelvic area among women. It is estimated that up to $70 \%$ of women develop UF during their fertile life [1]. Black women have the highest risk to develop UF. Hormone concentrations, growth factors and increased angiogenesis are thought to play an important role in the formation and growth of UF [2,3]. The use of oral contraceptives and high parity tend to lower the risk [4]. Approximately $25 \%$ of women with UF suffer from symptoms [5]. UF are associated with a high morbidity and may lead to career delay [4]. The main symptoms can be classified into three categories: heavy or prolonged menstrual bleeding, bulk-related symptoms or reproductive dysfunction and obstetric difficulties [6]. Symptoms can be assessed using the Uterine Fibroid Symptom-Quality of Life questionnaire (UFS-QoL), a validated disease-specific questionnaire including questions about symptom severity (transformed Symptom Severity Score; tSSS) and Quality of Life (QoL) (transformed Health-Related Quality of Life; tHRQL) [7].

Women suffering from symptomatic UF most commonly start with (hormonal) medication. When this is ineffective, more invasive treatment options such as the surgical interventions myomectomy or hysterectomy, are considered. Hysterectomy is the most frequently performed gynecological procedure worldwide, with UF being its most important indication [8,9]. Less invasive treatment options include Uterine Artery Embolization (UAE) and Magnetic Resonance-guided High-Intensity Focused Ultrasound (MR-HIFU) [10]. MR-HIFU is a non-invasive therapeutic modality which combines image guidance, using Magnetic Resonance Imaging (MRI), with a therapeutic High-Intensity Focused Ultrasound (HIFU) transducer [8].

Because not all fibroids are suitable for a minimally invasive fibroid therapy, an MRI is often performed to assess a patients' eligibility $[11,12]$. However, there is limited data about biological characteristics of uterine fibroids (UF) on the histological level and on MR imaging. Based on what is known about the histopathology, multiple subtypes of uterine fibroids are recognized [13]. Uterine fibroids are composed of smooth muscle fiber bundles, surrounded by collagenous fibrous connective tissue containing blood vessels. Also different types of mast cells are present. The density of each of these cell types is different for the various types of uterine fibroids. Because of these differences, fibroids react differently on thermal ablation. At this moment, there are no perfect imaging classifiers available to predict treatment outcome or to determine the optimal treatment modality for the individual patient.

The most commonly used classification system to predict the ultrasonic heating efficacy of UF is the Funaki classification. The Funaki classification divides fibroids into three subgroups based on the Signal Intensity (SI) of UF on T2-weighted (T2w) MR images compared to the SI of muscle and myometrium [14, 15]. Funaki type 3 fibroids can be unresponsive to MR-HIFU therapy, so are generally considered ineligible [12]. Funaki type 1 fibroids are expected to be good candidates for MR-HIFU treatment whereas almost all Funaki type 2 can be treated successfully [14], but varying treatment results have been reported $[16,17]$. This inaccuracy hampers the potential impact of MR HIFU treatment in UF patients. Therefore, the search for more discriminative parameters has started and further explorative research about biological characteristics of UF on MRI is needed.

To select patients for UAE, Kang et al. defined another screening tool based on T2w images, the so called Scaled Signal Intensity $[18,19]$. In contrast to the Funaki classification, the SSI score has a continuous numeric scale, and may, therefore, be more appropriate for screening when using a multiparametric MRI approach. To calculate the SSI the SI of a UF is scaled between 0 and 100 using the intensities of the rectus abdominis muscle and of subcutaneous fat as the minimum and maximum reference values for the scale, respectively. UF with a high SI on T2WI are more suitable for UAE. The SSI cutoff value to predict a volume reduction rate $>50 \%$ after UAE was reported to be $>18.16$ [19]. Park et al. [18] observed a correlation between SSI and MR-HIFU treatment result, e.g. the non-perfused volume (NPV) percentage. The SSI cutoff value to predict NPV $>45 \%$ was $<16.0$, thus MRHIFU is more effective in UF with a low SI on T2WI [18, 19]. This difference between MR-HIFU and UAE might be due to the fact that fibroids with a high SI on T2WI are histopathologically correlated with high vascularity and proliferative activity which are both associated with a poor MR-HIFU treatment outcome.

In the past other, more quantitative, approaches have been explored to improve the screening of patients leading to improved therapy outcomes of MR-HIFU ablation of UFs.

A quantitative parameter that could be of interest for screening is the transverse relaxation time of the tissue (T2), which can be mapped using a T2-mapping pulse sequence with appropriate post-processing. Compared to T2w scans, T2-mapping has the benefit of being more independent of MRI settings and hardware imperfections [20], and does not require a reference tissue. Therefore, T2-mapping could be more useful for classification. It is expected that the values of the T2 relaxation time will reflect relevant UF tissue properties, in particular blood volume and interstitial fluid volume. Histopathologically, UF with a long T2 relaxation time are correlated with higher water content, e.g. interstitial fluid or blood volume. These features are both associated with poor therapeutic efficacy of MR-HIFU. Additionally, 
it is difficult to elevate the temperature in high perfused tissues [12].

In the current study, we also evaluated different combinations of $b$-values to investigate if the characterization of UF tissue can be refined by adding information obtained from the apparent diffusion coefficient (ADC) maps. Andrews et al. [29] reported that Funaki type 3 fibroids have higher ADC values compared to Funaki type 1 and 2 fibroids, which is indicative of increased extracellular water content and/or blood volume. Ikink et al. [21] have demonstrated that the ADC in fibroid tissue is influenced by the choice of $b$-values and can contain information about the diffusion as well as the perfusion/blood volume in the UF. A significant decrease was seen in ADC values with the lowest $b$-values $(0$ and 200) between the ablated UF volume pre and post MRHIFU treatment. An explanation for these observations may be that the ADC acquired for low $b$-values strongly reflects perfusion effects, i.e. the micro-circulation of blood in the capillaries of the UF [21]. Moreover, Funaki type 3 fibroids demonstrate a higher pseudo diffusion coefficient compared to type 1 and 2 which could be related to higher vascularity [29]. Therefore, ADC might be useful to characterize UF tissue and to be able to distinguish between the different UF subtypes.

To possibly further characterize UF tissues, long-echo time (TE) ADC using low $b$-values was also investigated to emphasize not only the low blood perfusion but also the T2 relaxation time. The amount of water is also expected to be different between leiomyoma subtypes. Therefore, a better characterization of UF tissues could be provided by setting a longer echo time for the acquisition.

\section{Materials and methods}

This single-center, prospective, explorative study was performed at the Gynecology and Radiology departments of Isala Hospital, Zwolle, the Netherlands. This study was approved by the local ethics committee (registration number NL53499.075.15) and the hospital Board.

\section{Patients}

All patients presenting with UF symptoms at the gynecology outpatient clinic between December 2015 and June 2017 were evaluated for inclusion. Inclusion criteria were: UF confirmed on ultrasound, age over 18 years and pre- or peri-menopausal state. Exclusion criteria were: pregnancy, calcification of the UF, severe obesity or MRI contra-indications including contrast allergy. All eligible patients with symptomatic UF were offered to undergo an MRI examination regardless of their interest in MR-HIFU treatment. Potentially eligible patients for MR-HIFU therapy were counseled after the MRI. Results of the MR-HIFU treatments and post-treatment evaluation will be reported in a future paper. Informed consent was obtained before the MRI or filling out the UFS-QoL questionnaire. The tSSS and the tHRQL were calculated from the UFS-QoL questionnaire resulting in a scoring range between 0 and 100; higher scores indicate greater symptom severity or better QoL. Spies et al. [22] reported that fibroid patients at baseline scored 64.8 for the tSSS and 40.8 for the tHRQL and that the normal control group scored 15.3 for the tSSS and 92.8 for the tHRQL. Patient (age and ethnicity) and UF (number of UF, fibroid volume and location) characteristics were compared between the three Funaki types and related to outcomes of the UFS-QoL.

\section{Imaging protocol}

MRI scans were performed on a clinical 1.5-T MRI system (Achieva; Philips Healthcare, Best, the Netherlands). Patients were scanned in prone position. Sequence parameter settings are listed in Table 1. The examination was started with a survey followed by a sagittal T2w multishot Turbo Spin Echo (TSE) (TSE factor 17; TE $125 \mathrm{~ms}$ ) and an axial T2w multishot TSE (TSE factor 18; TE $110 \mathrm{~ms}$ ) to identify anatomical structures within the pelvis. After administration of a contrast agent (DOTAREM ${ }^{\circledR}, 0.2 \mathrm{~mL} / \mathrm{kg}$; Gadoterate Meglumine, $0.1 \mathrm{mmol} / \mathrm{kg}$; Guerbet; Aulnay-sous-Bois, France) three-dimensional (3D) T1-weighted (T1W) spoiled gradient echo (FFE) images were obtained (Table 1). Contrast-Enhanced (CE) images were used to assess the viability of the UF. Furthermore, the study protocol consisted of short-TE (64 ms) DWI and long-TE (140 ms) DWI series using a fat-suppressed multi-slice single-shot spin echo-echo planar imaging (SE-EPI) sequence (Table 1). To reduce the scan time, the multi-slice multi-echo sequence, T2-mapping, was performed using a multishot gradient and spin echo (GRASE) sequence with fat-suppression (Table 1). Twelve echoes were acquired, with TE ranging from $20-240 \mathrm{~ms}$ in $20 \mathrm{~ms}$ increments. The quantitative imaging protocol was obtained before the contrast administration. The total acquisition time of the MRI protocol was approximately $20 \mathrm{~min}$.

\section{Generation of parameter maps}

T2-mapping: T2 maps were generated by voxel-wise fitting of a mono-exponential function to the pixel signal intensity $\mathrm{S}$ for the different TEs, with $\mathrm{T} 2$ and $S_{0}$ as a fit parameter:

$S\left(T_{E}\right)=S_{0} \times e^{-T_{E} / T_{2}}$

The first echo was omitted from the fit since it has a stimulated echo component in it [23]. 
Table 1 The MRI protocol with relevant imaging parameter settings

\begin{tabular}{|c|c|c|c|c|c|c|}
\hline & $\mathrm{T} 2 \mathrm{w}$ axial & $\mathrm{T} 2 \mathrm{w}$ sagittal & T2-mapping & DWI & DWI long TE & CE-T1w \\
\hline Scan type & $\begin{array}{l}\text { TSE multishot } \\
\text { TSE factor } 18\end{array}$ & $\begin{array}{l}\text { TSE multishot } \\
\text { TSE factor } 17\end{array}$ & $\begin{array}{l}\text { GRASE multishot } \\
\text { TSE factor } 12 \\
\text { EPI factor } 5\end{array}$ & $\begin{array}{l}\text { Single shot SE-EPI } \\
\text { EPI factor } 51\end{array}$ & $\begin{array}{l}\text { Single shot SE-EPI } \\
\text { EPI factor } 51\end{array}$ & $\begin{array}{l}\text { 3D FFE } \\
\text { TFE multishot } \\
\text { TFE factor } 44\end{array}$ \\
\hline$T_{E}(\mathrm{~ms})$ & 110 & 125 & $n \times 20$ & 64 & 140 & 2.6 \\
\hline$T_{R}(\mathrm{~ms})$ & 3656 & 6219 & 2438 & 2673 & 6715 & 5.4 \\
\hline Flip angle $\left(^{\circ}\right)$ & 90 & 90 & 90 & 90 & 90 & 10 \\
\hline Slice thickness $(\mathrm{mm})$ & 3.5 & 3.0 & 7.0 & 7.0 & 7.0 & 3.0 \\
\hline ACQ Matrix & $356 \times 198$ & $356 \times 187$ & $112 \times 82$ & $112 \times 80$ & $112 \times 80$ & $168 \times 157$ \\
\hline FOV $(\mathrm{mm})$ & $250 \times 180$ & $250 \times 180$ & $250 \times 188$ & $250 \times 188$ & $250 \times 188$ & $250 \times 250$ \\
\hline ACQ Voxel size (mm) & $0.7 \times 0.88$ & $0.7 \times 0.94$ & $2.23 \times 2.23$ & $2.23 \times 2.26$ & $2.23 \times 2.26$ & $1.5 \times 1.49$ \\
\hline Scan $\%$ & 77.3 & 73.0 & 98.2 & 95.6 & 95.6 & 94 \\
\hline NSA & 2 & 3 & 2 & 4 & 4 & 3 \\
\hline Half scan & No & No & No & Yes & Yes & No \\
\hline Fat suppression & No & No & SPIR & SPAIR & SPAIR & SPAIR \\
\hline Scan duration (min:s) & $2: 48.2$ & $3: 31.5$ & $2: 55.5$ & $6: 51.6$ & $4: 35.3$ & $2: 31.2$ \\
\hline
\end{tabular}

$D W I$ diffusion weighted imaging, $T_{E}$ echo time, TSE turbo spin echo, GRASE gradient and spin echo, EPI echo planar imaging, $S E$ spin echo, $F F E$ fast field echo, TFE turbo field echo, $T_{R}$ repetition time, $A C Q$ acquired, FOV field of view, Scan $\%$ scan percentage, NSA number of signal averages, $S P I R$ spectral presaturation inversion recovery, SPAIR spectral selection attenuated inversion recovery

$D W I$ DWI images were acquired with $7 b$-values: 0 , $50,100,200,400,600$ and $800 \mathrm{~s} / \mathrm{mm}^{2}$. ADC maps from the DWI images were calculated by a voxelwise fit of a mono-exponential function to the pixel signal intensities $(S)$ using the formula, with ADC and $S_{0}$ as fit parameters:

$$
S(b)=S_{0} \times e^{-b \times \mathrm{ADC}}
$$

Three different ADC maps were calculated. The first ADC map was reconstructed with all measured $b$-values, the second ADC map with only the three lowest $b$-values $(0,50$, $100 \mathrm{~s} / \mathrm{mm}^{2}$ ) and the third ADC map with only the three high $b$-values $\left(400,600,800 \mathrm{~s} / \mathrm{mm}^{2}\right)$. Four different $b$-values $(0$, $50,100,200 \mathrm{~s} / \mathrm{mm}^{2}$ ) were acquired for the long-TE DWI sequence and the ADC map was derived using these low $b$-values.

\section{Measurements}

IntelliSpace Portal (ISP) software (version 8, Philips Healthcare, Best, The Netherlands) was used for calculating parameter maps and for the region-of-interest (ROI) analysis. In the case of multiple UFs, the largest UF was chosen for analysis. The total UF volume was measured by drawing a Volume Of Interest (VOI) on the T2w images using the tumor tracking application in ISP. A user-selected seed location was chosen at an initial slice, approximately in the center of the UF. Then, the system measured UF volume automatically and the observer manually adapted the segmentation when necessary. The following ROI placement protocol (Fig. 1) was used to determine the Funaki classification and SSI: (1) all measurements were performed on the axial slice

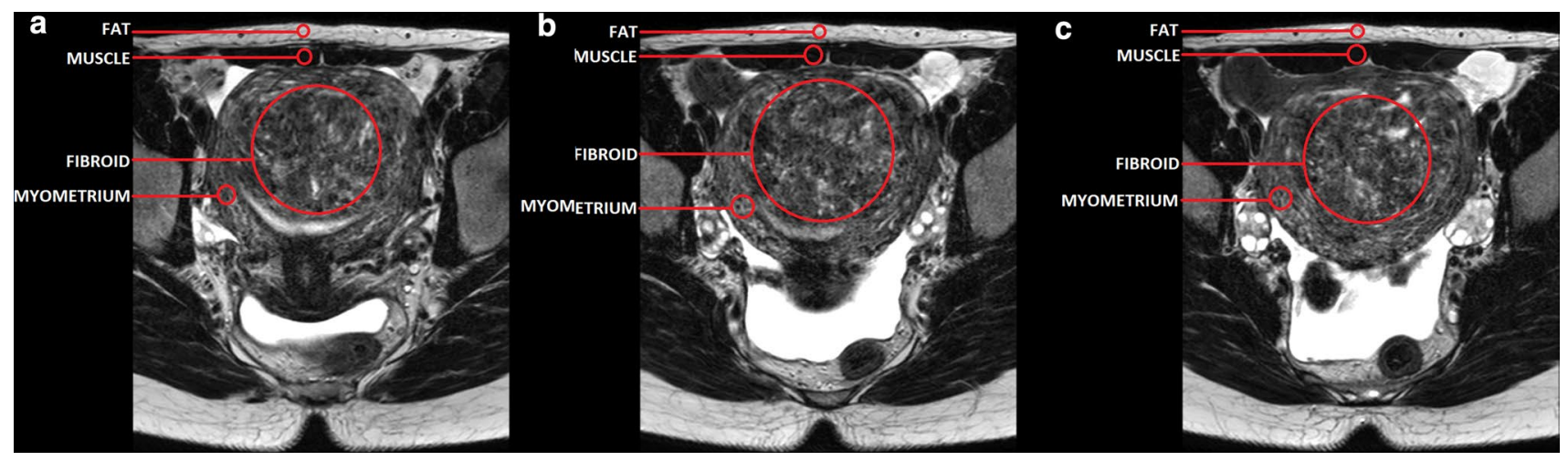

Fig. 1 The ROI placement protocol used to determine the Funaki class and SSI of the uterine fibroid. All measurements were performed manually on the axial slice of the $\mathrm{T} 2 \mathrm{w}$ series in $2 \mathrm{D}$ and were repeated three times. The ROI size was maximum while avoiding adjacent structures 
of the T2w series (TE $110 \mathrm{~ms}$ ); (2) the circular ROI's were manually drawn three times in $2 \mathrm{D}$; (3) the median value was used for classification; (4) the ROI size was maximum while avoiding adjacent structures; (5) anatomical locations of the ROI's were predefined, the UF tissue ROI ( $\mathrm{SI}_{\text {fibroid }}$ ) was placed approximately in the center, the muscle ROI in the rectus abdominis muscle ( $\mathrm{SI}_{\text {muscle }}$ ), the fat ROI in the subcutaneous abdominal fat $\left(\mathrm{SI}_{\mathrm{fat}}\right)$ and the myometrium ROI ( $\left.\mathrm{SI}_{\text {myometrium }}\right)$ not close to the cervix, UF or endometrium.

After all ROI's were drawn, the UF was classified as a Funaki type 1,2 or 3 according to the following criteria: (1) SI equal to or lower than the SI of skeletal muscle; (2) SI higher than the SI of skeletal muscle, but lower than the SI of myometrium; (3) SI comparable to or higher than the SI of myometrium, respectively.

The SSI score was calculated with the following formula:

$\mathrm{SSI}=\frac{\mathrm{SI}_{\text {fibroid }}-\mathrm{SI}_{\text {muscle }}}{\mathrm{SI}_{\text {fat }}-\mathrm{SI}_{\text {muscle }}} \times 100$

Finally, the SSI score was related to the Funaki classification. Furthermore, since Funaki's method does not prescribe the amount of $\mathrm{T} 2$-weighting required, the currently used Funaki classification was reevaluated by comparing the Funaki distribution for $\mathrm{T} 2 \mathrm{w}$ scans with four different echo times. All ROI's were drawn on the T2w images with TE $110 \mathrm{~ms}$ as well as on the T2w images with $125 \mathrm{~ms}$ and in the images of the corresponding echoes of the TSE sequence from the T2-mapping (TE $20 \mathrm{~ms}$ and $60 \mathrm{~ms}$ ). Thus, each UF was classified four times. The Funaki distribution measured on $110 \mathrm{~ms}$ was used for analysis since this is the standard TE in our local MRI protocol.

All T2 and ADC maps were evaluated to distinguish UF tissue from myometrium. On these maps, ROI's were placed in the UF (center) and the uterine myometrium on the axial slices. Firstly, the ROI's were drawn on the T2 and then copied onto the ADC maps with different $b$-value combinations and different echo times. The conventional $\mathrm{T} 2 \mathrm{w}$ scans were used as a visual reference for the identification of UF tissue and myometrium. The measured T2 and ADC values were compared between the three Funaki types and related to the SSI score of the UF.

\section{Statistical analysis}

To minimize random errors, all ROI measurements were performed three times by two observers. Per observer the median value was used for further analysis. To minimize the inter-observer variability, the ROI placement approach was standardized in the protocol as outlined above. All VOI, ROI data and mean values were stored in an Excel database (Microsoft). Statistical analyses were performed using IBM
SPSS version 25 and R, version 3.4.2, the R Foundation for Statistical Computing. Categorical data were presented as $n$ $(\%)$. Continuous variables were presented as mean $( \pm \mathrm{SD})$ in the case of a normal distribution, in the case of a skewed distribution the median $(Q 1-Q 3)$ values were also reported. Distribution was assessed by the Shapiro-Wilk test, complemented by plots and the indices skewness and kurtosis. Differences between MR-parameters of UF tissue and myometrium were tested by means of the paired $T$-test or the Wilcoxon's signed-rank test. Correlations between continuous variables (SSI score, MR-parameters, tSSS, tHRQL, age, UF volume) were investigated using Pearson's/Spearman's correlation. Additionally, to investigate categorical independent variables (Funaki classification and intra-uterine location of the UF) regression analysis was performed and dummy variables were used.

\section{Results}

In total, 87 women with symptomatic UF were included in this study (Table 2). All included patients underwent an MRI examination and a typical example of the acquired MR images are shown in Fig. 2. Median age was 46.0 (42.0-49.0) years and the majority of the study participants were Dutch (73.6\%). Most women had multiple UF (58.6\%) with a median volume of $115.3 \mathrm{~mL}$ (31.2-248.3). Intramural location of the UF was frequent (56.3\%) compared to a submucosal (21.8\%) or subserosal location of the UF (21.8\%).

\section{Funaki}

Overall, $66.7 \%$ of the UFs were classified as Funaki 2 (5.7\% as type 1 and $27.6 \%$ as type 3 ) when measured on the axial T2w images (TE $110 \mathrm{~ms}$ ). The distributions of the UF over the three Funaki types differed for the four TE (20 ms, $60 \mathrm{~ms}, 110 \mathrm{~ms}$ and $125 \mathrm{~ms}$ ) (Fig. 3). The shorter the echo time used to determine the Funaki classification the higher the percentage of Funaki type 3 fibroids. Moreover, no Funaki type 1 fibroids were found when the Funaki classification was based on a scan with TE $125 \mathrm{~ms}$. Several significant differences were found in patient and fibroid characteristics between the three Funaki types; the UF volume was significantly higher $(p=0.022)$ in the Funaki type 3 group (mean: $244.51 \mathrm{~mL}$ ) compared to the Funaki type 2 (mean: $143.14 \mathrm{~mL}$ ). The number of UFs was significantly higher $(p=0.032)$ in the group of patients with Funaki type 1 fibroids (60.00\% had more than 5 fibroids) than the patients in the Funaki type 3 group (58.33\% had only 1 UF). 
Table 2 Patient characteristics

\begin{tabular}{|c|c|}
\hline \multicolumn{2}{|l|}{ Characteristic } \\
\hline Age (years) ${ }^{\mathrm{a}}$ & $\begin{array}{l}46.0(42.0-49.0) \\
45.6 \pm 5.4\end{array}$ \\
\hline \multicolumn{2}{|l|}{ Number of fibroids ${ }^{b}$} \\
\hline 1 & $36(41.4)$ \\
\hline 2 & $14(16.1)$ \\
\hline 3 & $15(17.2)$ \\
\hline 4 & $4(4.6)$ \\
\hline 5 or more & $18(20.7)$ \\
\hline \multicolumn{2}{|l|}{ Fibroid location ${ }^{\mathrm{b}}$} \\
\hline Submucosal & $19(21.8)$ \\
\hline Intramural & $49(56.3)$ \\
\hline Subserosal & $19(21.8)$ \\
\hline Fibroid volume $(\mathrm{mL})^{\mathrm{a}}$ & $\begin{array}{l}115.3(31.2-248.3) \\
171.5 \pm 182.0\end{array}$ \\
\hline \multicolumn{2}{|l|}{ Funaki classification $^{\mathrm{b}}$} \\
\hline 1 & $5(5.7)$ \\
\hline 2 & $58(66.7)$ \\
\hline 3 & $24(27.6)$ \\
\hline $\operatorname{SSI}^{\mathrm{a}, \mathrm{c}}$ & $\begin{array}{l}12.7(6.3-25.1) \\
16.91 \pm 13.19\end{array}$ \\
\hline $\mathrm{tSSS}^{\mathrm{d}, \mathrm{e}}$ & $49.1 \pm 21.0$ \\
\hline $\mathrm{tHRQL}^{\mathrm{c}, \mathrm{c}}$ & $57.0 \pm 23.9$ \\
\hline \multicolumn{2}{|l|}{ Dutch ethnicity ${ }^{\mathrm{b}}$} \\
\hline Yes & $64(73.6)$ \\
\hline No & $23(26.4)$ \\
\hline
\end{tabular}

${ }^{a}$ Median (interquartile range) and mean \pm standard deviation

${ }^{\mathrm{b}} n(\%)$

${ }^{\mathrm{c}}$ Scaled signal intensity

${ }^{\mathrm{d}}$ Mean \pm standard deviation

${ }^{\mathrm{e}}$ Transformed symptom severity score

${ }^{\mathrm{f}}$ Transformed health-related quality of life

\section{SSI}

The average measured SSI-score was 12.7 (6.3-25.1). A significant difference was seen between Funaki type 3 compared to Funaki type $2(p<0.001)$ or Funaki type 1 $(p<0.001)$ and also between Funaki type 1 and Funaki type 2 fibroids $(p=0.001)$.

\section{Quantitative parameters}

Significant differences were found between the normal uterine myometrium and UF tissue for the measured T2 values, the long-TE ADC map with low $b$-values and two of the three ADC maps, namely, the map derived from all $b$-values and the map derived from only high $b$-values (Table 3 ). The ADC maps obtained with low $b$-values did not show a significant difference between UF and myometrium.
A significant difference in observed $\mathrm{T} 2$ values was seen between Funaki type 3 versus $1(p<0.001)$ or $2(p<0.001)$ and Funaki type 2 versus $1(p=0.005)$. The long-TE ADC with low $b$-values resulted in a significant difference between Funaki type 1 compared to Funaki type $2(p=0.016)$ and type 3 ( $p=0.006)$. Moreover, significant differences were also observed in the ADC with all $b$-values $(p=0.029)$ and ADC with high $b$-values $(p=0.025)$ between Funaki type 3 versus Funaki type 2.

The SSI and quantitative MR-parameters showed significant correlations between the SSI and T2-mapping $\left(p<0.001 ; \rho_{s}=0.82\right)$, ADC with all $b$-values $(p=0.004$; $\left.\rho_{s}=0.31\right)$, ADC with only high $b$-values $(p<0.001$; $\left.\rho_{s}=0.44\right)$ and long-TE ADC with low $b$-values $(p=0.004$; $\left.\rho_{s}=0.31\right)$.

Significant differences were found between the three Funaki types in SSI, ADC values and T2-mapping values. To possibly demonstrate these differences, the distribution of the three Funaki types for these MR-parameters is visualized in Fig. 4.

\section{UFS-QOL}

Patients had an average tSSS of $49.1 \pm 21.0$ before the MRI examination and an average tHRQL of $57.0 \pm 23.9$ (Table 2). As expected, the tSSS and tHRQL were strongly correlated $(p<0.001 ; r=-0.81)$. The tSSS showed significant differences between Funaki type 3 compared to Funaki type $2(p<0.001)$ and Funaki type $1(p=0.040)$ (Fig. 5). The tHRQL showed a significant difference $(p<0.001)$ between Funaki type 3 compared to Funaki type 2 (Fig. 5). Moreover, the tSSS was significantly different for ethnicity $(p=0.030)$ and intra-uterine location of the UF; tSSS was significantly different for subserosal located UF compared with intramural $(p=0.002)$ and submucosal UF $(p=0.001)$. Subsequently, the tHRQL was also significantly different for ethnicity ( $p=0.015)$ and the location of the UF; tHRQL was significantly different for subserosal located UF compared with intramural $(p=0.005)$ and submucosal UF $(p=0.001)$.

\section{Discussion}

In this study, an unselected patient population with UF underwent a multiparametric MRI examination to investigate differences between fibroid tissue types in T2 and ADC values and to compare the SSI score, patient characteristics and scores of the UFS-QoL between the three different Funaki types. We will discuss the investigated potentially discriminating parameters in detail below.

The three Funaki types showed differences in UF volume, indicating that Funaki type 3 fibroids are in general larger. Funaki type 1 fibroids are not only smaller on average, but 


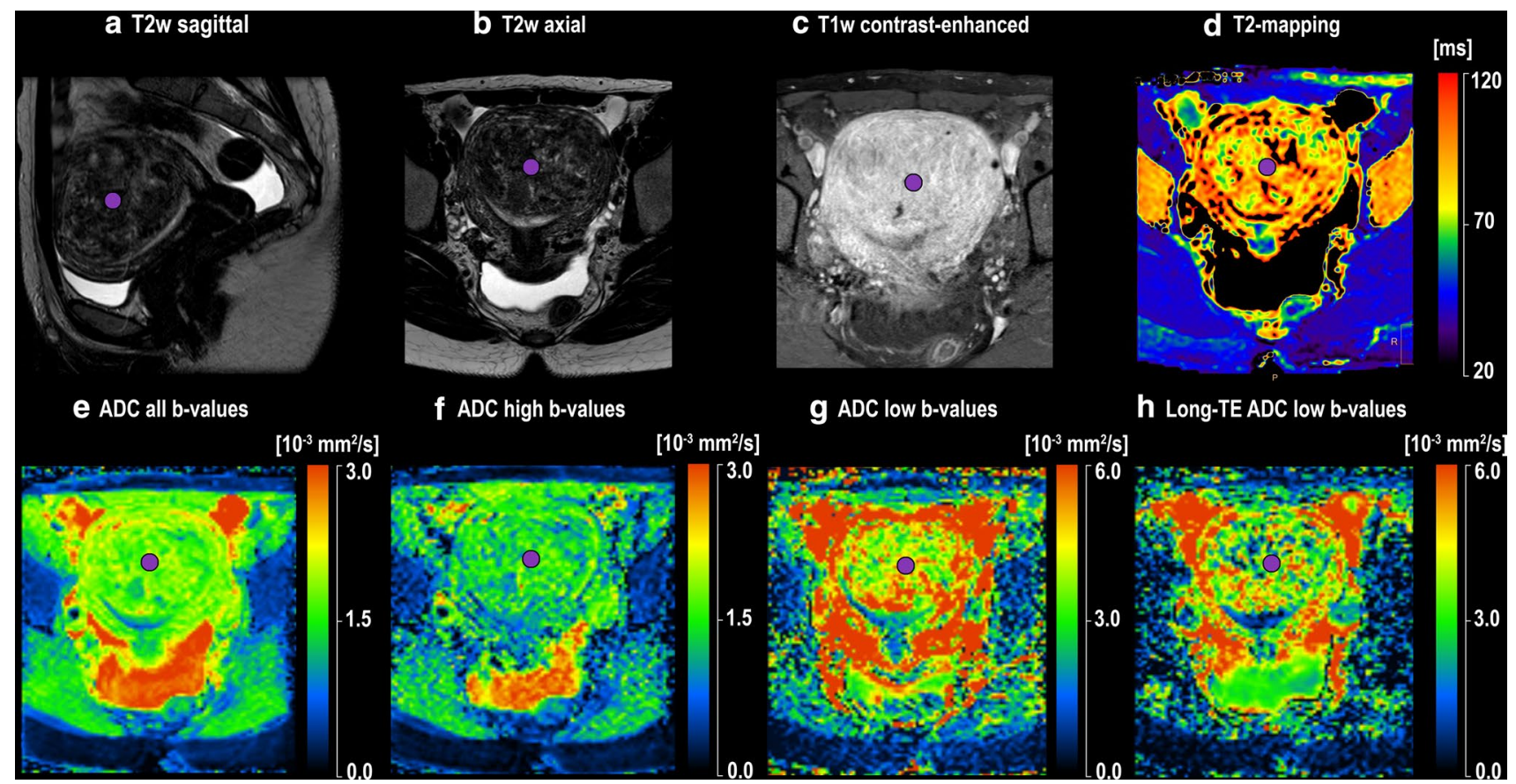

Fig. 2 Images of all the different MR-parameters used in the MRI protocol of this study. The purple dot represents the middle of the uterine fibroid. On the two left upper images, a single intramural uterine fibroid is shown with distortion of the uterine cavity. The signal intensity of the fibroid is lower than the uterine myometrium, but higher than the rectus abdominis muscle, so this is a Funaki type
2 fibroid. After administration of gadolinium on the T1-weighted image, there is homogeneous enhancement of the uterine fibroid which is less than the enhancement of the uterine wall (myometrium). The derived T2 map is shown on the right upper image. The reconstructed ADC maps with different combinations of $b$-values are demonstrated in the lower part of the image
Fig. 3 Barplot showing distribution of patients over the three Funaki types on T2-weighted images with four different echo times (TE). The number of patients is shown on the $Y$-axis and the Funaki classification on the $X$-axis. The four different echo times $(20 \mathrm{~ms}$, $60 \mathrm{~ms}, 110 \mathrm{~ms}$ and $125 \mathrm{~ms}$ ) are indicated by different colors

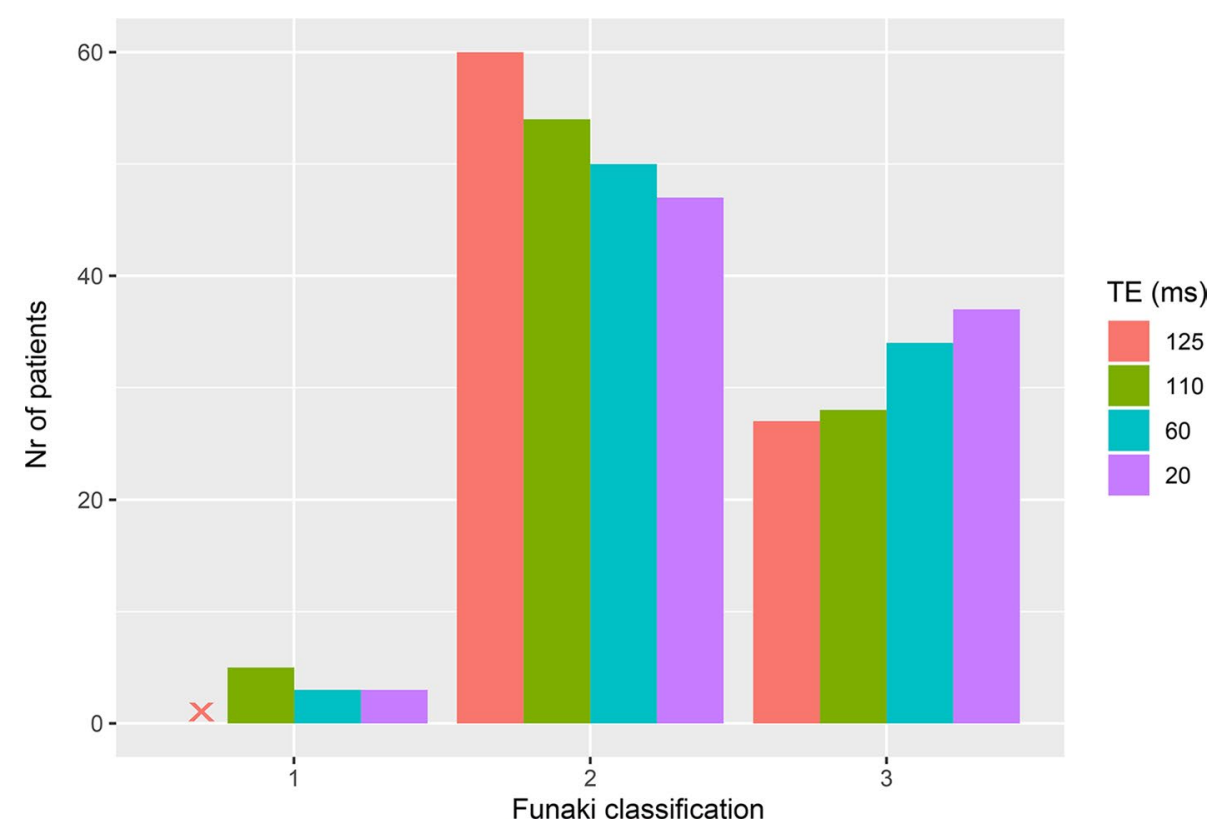

patients with Funaki type 1 fibroid also develop more UF compared to other Funaki types [24].

Importantly, Funaki and colleagues did not specify the scan protocol settings for the T2w images in the MRI protocol which they advised to use for their Funaki classification of UF. To investigate the effect of the strength of T2 weighting on the Funaki classification, we used different TE's in the T2w scans used to determine Funaki type. 
Table 3 Comparison of the observed $\mathrm{T} 2$ values and the ADC values $\left(10^{-3} \mathrm{~mm}^{2} / \mathrm{s}\right)$ calculated with different combinations of $b$-values between uterine fibroids and myometrium

\begin{tabular}{lllr}
\hline & Uterine fibroid & Myometrium & $p$ value \\
\hline T2-mapping $^{\mathrm{a}}$ & $70.60(58.70-84.50)$ & $78.30(69.30-86.40)$ & $0.001^{\mathrm{b}}$ \\
$b$-values 0, 50, 100, 200, 400,600,800 & $1.21 \pm 0.24$ & $1.39 \pm 0.25$ & $<0.001^{\mathrm{d}}$ \\
$b$-values 0, 50, 100 & $3.18 \pm 0.84$ & $3.39 \pm 1.01$ & $0.110^{\mathrm{d}}$ \\
$b$-values 400, 600, 800 & $1.00(0.90-1.10)$ & $1.10(1.00-1.20)$ & $<0.001^{\mathrm{b}}$ \\
Long-TE ADC $b$-values 0, 50,100, 200 & $2.49 \pm 0.66$ & $2.88 \pm 0.93$ & $0.002^{\mathrm{d}}$ \\
\hline
\end{tabular}

${ }^{\mathrm{a}}$ Median (interquartile range)

${ }^{\mathrm{b}}$ Calculated using the Wilcoxon Signed Rank Test

${ }^{\mathrm{c}}$ Mean \pm standard deviation

${ }^{\mathrm{d}}$ Calculated using the paired $t$-test
Fig. 4 Pairs plot for correlation of descriptive variables and Scaled Signal Intensity (SSI) split by Funaki classification. Pairs plot is a combination of scatter plots, density functions and correlation for each Funaki Class. The different Funaki groups are shown by different colors. On the $Y$-axis, the variables are the T2-mapping values, the ADC with all $b$-values and the SSI

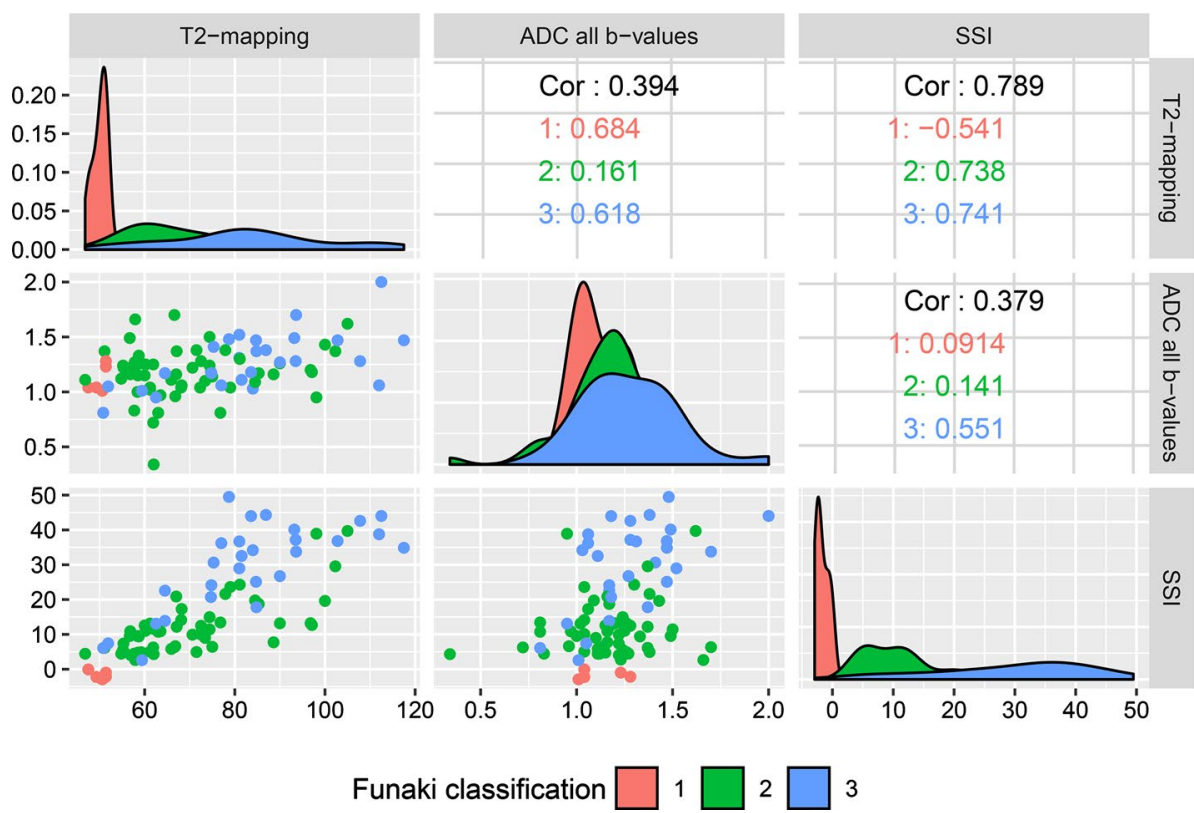

The distribution of the Funaki classification was different depending on the echo time used which is important to realize. When using the $20 \mathrm{~ms}$ and $60 \mathrm{~ms} \mathrm{~T} 2 \mathrm{w}$ images, more fibroids were classified as a Funaki type 3. This can be explained by the fact that the SI of myometrium and UF tissue on $\mathrm{T} 2 \mathrm{w}$ images is more similar when the TE is shorter. In addition, the MRI scan must be of good diagnostic quality to evaluate anatomy and to detect incidental findings. Therefore, the T2w images with a TE of $20 \mathrm{~ms}$ or $60 \mathrm{~ms}$ are not applicable for screening. When using a TE of $125 \mathrm{~ms}$, we did not find any Funaki type 1 fibroids. This can be explained by the fact that fibroids contain more water (in particular blood volume) than muscle, so the SI of muscle on $\mathrm{T} 2 \mathrm{w}$ images with TE of $125 \mathrm{~ms}$ is lower than the SI of UF. Moreover, less Funaki type 3 fibroids were seen with TE $125 \mathrm{~ms}$ and more Funaki type 2 fibroid than with the other TE setting. The Funaki distribution seen at T2w images with $125 \mathrm{~ms}$ might lead to a higher treatment failure rate due to allocation of UF to the Funaki type 2 group instead of Funaki type 3 group since it is known that treatment failures still occur in the Funaki type 2 group [17, 25]. The true clinical impact of these findings should be investigated in the future, but it should be noted that the TE in the MRI protocol for T2w images greatly influences the distribution of the different Funaki groups. Furthermore, a standardized approach for the ROI-based measurements to determine Funaki has not been established yet. We propose a standardized ROI placement approach as described in our methods section because random measurements are prone to selection bias. In clinical practice, to determine the Funaki class of a UF is often performed by subjective visual assessment of the radiologist with an even higher risk for inaccuracy. Due to the heterogeneity of the SI of the UF on T2w images, a UF could be allocated to the Funaki type 2 group instead of the Funaki type 3 group.

Our findings provide additional insights into the suboptimal nature of the Funaki classification as a discriminator and tissue characterization is further limited because only three subclasses exist. The SSI, on the other hand, is a numeric variable and thus may be a more suitable tool for patient 


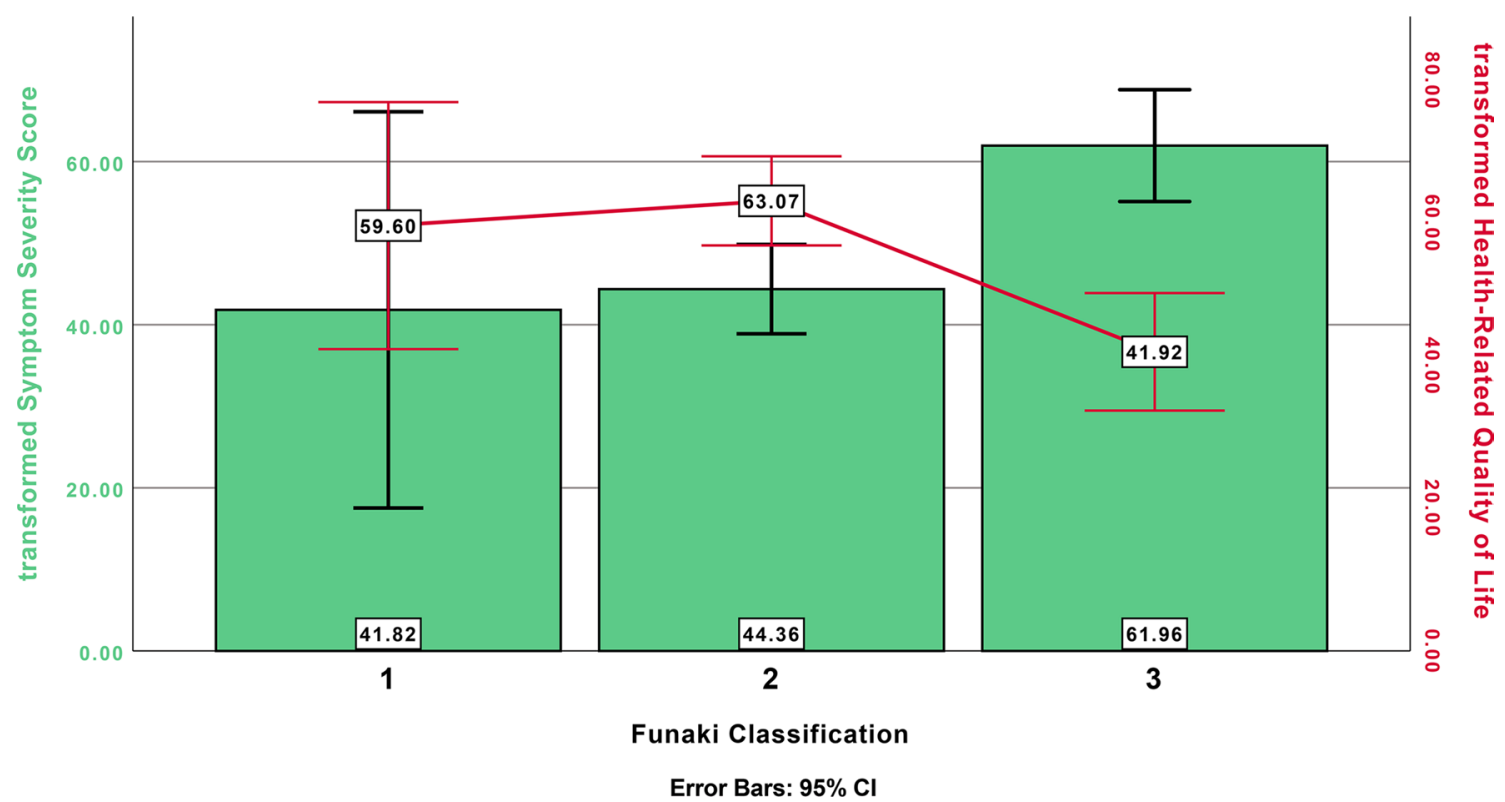

Fig. 5 Barplot demonstrating the relationship between the Uterine Fibroid Symptom-Quality of life (UFS-QoL) questionnaire. The Funaki classification is shown on the categorical $\mathrm{X}$-axis with the UFS-QoL on the dual $Y$-axes. The transformed Symptom Severity

selection when using a multiparametric MRI approach. Park et al. [18] noted that patients with a SSI-score of 16 or below had the highest change of an NPV $>45 \%$ after MR-HIFU treatment. When the SSI classification system described by Park et al. would have been used to determine suitability for MR-HIFU, only 52 patients would have been eligible. When considering Funaki type 1 and 2 eligible for treatment, 63 of our 87 patients would have been suitable for MR-HIFU treatment [14]. When Funaki classification is used, more patients are eligible for MR-HIFU compared to SSI.

Significant differences between myometrium and UF tissue were found in all the parametric MR images, except the ADC with low $b$-values. The T2 value in the UF was considerably lower than the $\mathrm{T} 2$ value of the myometrium, presumably due to less hydration of UF tissue compared to myometrium [26]. This finding corresponds with the finding that the ADC values are higher for myometrium than UF tissue. However, the ADC map with low $b$-values is not useful in differentiating between UF tissue and myometrium indicating that diffusion/perfusion characteristics of myometrium and UF tissue were similar. Ikink et al. [21] suggested that ADC maps obtained with only low $b$-values emphasizes the perfusion contribution to the ADC.

To differentiate UF types based on their diffusion/perfusion characteristics rather than on T2w images only, correlations with the SSI and Funaki classification were investigated. From all the combinations of ADC maps with different $b$-values, only the ADC map with the low $b$-values did not correlate with SSI. This could be explained by the
Score on the left $Y$-axis is depicted with green bars, the right $Y$-axis and red line represent the transformed Health-Related Quality of Life. Data are presented in mean for each Funaki category including error bars $(95 \% \mathrm{CI})$

fact the ADC map with low $b$-values reflects tissue perfusion [21], and movement of water molecules is not detected with T2 w imaging on which the SSI is measured. The Funaki type 3 UF showed a higher long-TE ADC value than a Funaki type 1 , this could be expected since Funaki type 3 fibroids have a higher vascularity and an atypical Funaki type 3 UF could mimic leiomyosarcoma. Long-TE DWI can also be used to differentiate between malignant (high vascularity) and benign lesions, as demonstrated in previous studies, thus it might be a helpful screening tool to differentiate between UF tissues [27, 28]. Furthermore, significant differences were also seen between the Funaki classification and the ADC high $b$-values and ADC all-values. The finding that Funaki differs with the ADC map with all $b$-values is in line with Andrews et al. [29] who also suggested that Funaki type 3 fibroids exhibit higher ADC values than Funaki type 1 and 2, which implies increased extracellular water content and/ or blood volume in Funaki type 3 fibroids.

Quantitative T2-mapping was used to quantify UF tissue properties, in particular water content (including blood). Significant differences were found in observed T2 values between the three Funaki types and a strong correlation was found between the T2-mapping and SSI. Since all measurements are based on $\mathrm{T} 2 \mathrm{w}$ images these findings were to be expected. The findings in this study may indicate that both SSI and T2-mapping are useful parameters for the evaluation of UF tissue imaging features. The accuracy of the Funaki classification system is not sufficient to predict MR-HIFU treatment outcome [30]. A perfect classifier would correctly 
select treatable fibroids only based on their MR characteristics. Presumably, a multiparametric analysis would provide a more reliable prediction model for MR-HIFU treatment outcome. To optimize patient selection for MR-HIFU therapy, the screening SSI and T2-mapping values of the UF should be linked to treatment parameters to determine a cut-off value (as part of a prediction model). A previous study already suggested that T2-mapping could be the most valuable parameter for treatment outcome [31].

In general, patients with a lower tSSS had a higher tHRQL as expected [7]. Interestingly, tSSS outcomes of patients with Funaki type 3 UF were significantly higher than tSSS values of patients with Funaki type 1 and type 2 UF. In parallel, the tHRQL was significantly lower in patients with a Funaki type 3 UF compared to patients with a Funaki type 1 or 2 UF. One could argue that Funaki type 3 fibroids generally grow faster due to their hypervascularity which could lead to a more immediate onset of symptoms and, therefore, impact on UFS-QoL questionnaire which only considers symptoms during the previous 3 months [7]. Moreover, as mentioned earlier the UF volume is also higher compared to that of Funaki type 1 and type 2 UFs, which contributes to the tSSS. Furthermore, Dutch patients had a lower tSSS and an improved tHRQL compared to women with different ethnicity. This may be explained by cultural differences in self-perception [9]. A submucosal location of the UF increased the tSSS and lowered the QoL. This outcome is not surprising since a submucosal location of a UF leads to heavier bleeding and the tSSS part of the UFS-QoL questionnaire consists of eight questions in total from which four questions are related to bleeding symptoms.

A strength of this study was that an unselected patient population of women suffering from UF was included in this study independent of interest in MR-HIFU treatment. This led to a heterogeneous UF patient population, reducing the risk of selection bias. Furthermore, to the best of our knowledge this is the first in vivo study to investigate the imaging features of the total UF patient population and associate these UF characteristics with patient characteristics, symptom severity and QoL. However, the skewed distribution of the patients in the Funaki classification together with the small sample size of the study resulted in statistical difficulties and, therefore, the outcomes of the Funaki type 1 group should be interpreted with caution. The skewed distributions are most likely explained by the fact that some leiomyoma histological variants are more common than other subtypes [13]. We were unable to compare our findings with histology data because biopsies are not routinely performed to confirm the diagnosis of UF. Due to the explorative nature of this study, it seemed unethical to obtain pathological data, although future research to compare pre-treatment MR imaging with post-hysterectomy data would be valuable. Another limitation of this study was that the quality of some of the images was rather poor due to extrinsic (bowel or patient movement) reasons. Motion artefacts in the images made it more complicated to draw the ROI precisely. All ROI measurements were performed three times per observer for each tissue to minimize ROI placement errors. Often an outlier was seen in one of the ROI measurements and this outlier was eliminated using the median value for analysis. Furthermore, when performing the ROI measurements, it was difficult to standardize the measurement of the uterine myometrium since the location of the UF influenced where the ROI in the myometrium could be placed. No agreements could be made in advance about the specific location for the ROI placement in the myometrium.

Further cluster analysis using a larger sample size (with a larger Funaki type 1 group) could reveal if a more objective way than the Funaki classification can be found to refine the characterization of UF. Moreover, to optimize patient selection for minimally-invasive treatment options of UF the predictive value of the quantitative MR-parameters should be correlated to treatment outcome such as NPV\% and clinical follow-up.

\section{Conclusions}

This study showed that quantitative multiparametric MRI can differentiate between myometrium and UF tissue in an unselected patient population with symptomatic UF. Between different UF tissues, a large variation in morphologic characteristics was found with the quantitative MR-parameters. The SSI and T2-mapping values appeared to be useful parameters for the evaluation of UF tissue imaging features, however, these results should be linked to treatment outcome.

Acknowledgements This research was made possible with financial support from the Innovation and Science fund of Isala Hospital in Zwolle, the Netherlands.

Author contributions IV contributed to the study design, in the acquisition of data and data analysis, interpretation of data and writing of the manuscript. KJ had a substantial contribution in the acquisition of data, data analysis, interpretation of data and writing of the manuscript. $\mathrm{EH}$ had a substantial contribution to the study design, implementing the MRI study protocol and interpretation of the data. ME, PC and IN were involved in the analysis and the interpretation of data. JD and JS had major contributions to this study by including the study participants and facilitating the collection of data. $\mathrm{LB}, \mathrm{CM}$ and $\mathrm{AF}$ were involved in the interpretation of data and drafting the manuscript. $\mathrm{MB}$ was the principal investigator of this study and responsible for the conceptual design of the study, obtaining ethical approval and interpretation of the data. All authors critically revised the manuscript for important intellectual content and approved the final manuscript. 


\section{Compliance with ethical standards}

Conflict of interest Authors E. Heijman and P.C. Vos are both employees of Philips who have developed the MR-HIFU equipment. All other authors had no financial interest or conflicts of interest in the subject matter discussed in the submitted manuscript. All authors state that this study complies with the Declaration of Helsinki.

Ethical approval All authors state that this study complies with the Declaration of Helsinki.

\section{References}

1. Cramer S (1990) The frequency of uterine leiomyomas. Am J Clin Pathol 94:435-438

2. Stewart EA (2001) Uterine fibroids. Lancet (London, England) 357:293-298

3. Stewart EA, Laughlin-Tommaso SK, Catherino WH, Lalitkumar S, Gupta D, Vollenhoven B (2016) Uterine fibroids. Nat Rev Dis Primers 2:16043. https://doi.org/10.1038/nrdp.2016.43

4. Borah BJ, Nicholson WK, Bradley L, Stewart EA (2013) The impact of uterine leiomyomas: a national survey of affected women. Obstet Gynecol 209:1-319. https://doi.org/10.1016/j. ajog.2013.07.017

5. Buttram V (1981) Uterine leiomyomata: etiology, symptomatology, and management. Fertil Steril 36:433-445

6. Stewart E, Cookson C, Gandolfo R, Schulze-Rath R (2017) Epidemiology of uterine fibroids: a systematic review. BJOG 124:15011512. https://doi.org/10.1111/1471-0528.14640

7. Spies JB, Coyne K, Guaou Guaou N, Boyle D, Skyrnarz-Murphy K, Gonzalves SM (2002) The UFS-QOL, a new disease-specific symptom and health-related quality of life questionnaire for leiomyomata. Obstet Gynecol 99:290-300

8. Pron G (2015) Magnetic resonance-guided high-intensity focused ultrasound (MRgHIFU) treatment of symptomatic uterine fibroids: an evidence-based analysis. Ont Health Technol Assess Ser 15:1-86

9. Chen R, Keserci B, Bi H, Han X, Wang X, Bai W, Wang Y, Yang X, Yang J, Wei J, Seppala M, Viitala A, Liao Q (2016) The safety and effectiveness of volumetric magnetic resonance-guided highintensity focused ultrasound treatment of symptomatic uterine fibroids: early clinical experience in China. J Ther Ultrasound 4:27-016. https://doi.org/10.1186/s40349-016-0072-9

10. Ravina JH, Herbreteau D, Ciraru-Vigneron N, Bouret JM, Houdart E, Aymard A, Merland JJ (1995) Arterial embolisation to treat uterine myomata. Lancet (London, England) 346:671-672

11. Tempany CM, Stewart EA, McDannold N, Quade BJ, Jolesz FA, Hynynen K (2003) MR imaging-guided focused ultrasound surgery of uterine leiomyomas: a feasibility study. Radiology 226:897-905

12. Yoon SW, Lee C, Kim KA, Kim SH (2010) Contrast-enhanced dynamic mr imaging of uterine fibroids as a potential predictor of patient eligibility for mr guided focused ultrasound (MRgFUS) treatment for symptomatic uterine fibroids. Obstet Gynecol Int. https://doi.org/10.1155/2010/834275

13. Arleo EK, Schwartz PE, Hui P, McCarthy S (2015) Review of leiomyoma variants. AJR Am J Roentgenol 205:912-921. https ://doi.org/10.2214/AJR.14.13946

14. Funaki K, Fukunishi H, Funaki T, Sawada K, Kaji Y, Maruo T (2007) Magnetic resonance-guided focused ultrasound surgery for uterine fibroids: relationship between the therapeutic effects and signal intensity of preexisting T2-weighted magnetic resonance images. Am J Obstet Gynecol 196:184.e1-184.e6

15. Zhao WP, Chen JY, Chen WZ (2015) Effect of biological characteristics of different types of uterine fibroids, as assessed with T2-weighted magnetic resonance imaging, on ultrasound-guided high-intensity focused ultrasound ablation. Ultrasound Med Biol 41:423-431. https://doi.org/10.1016/j.ultrasmedbio.2014.09.022

16. Mindjuk I, Trumm CG, Herzog P, Stahl R, Matzko M (2015) MRI predictors of clinical success in MR-guided focused ultrasound (MRgFUS) treatments of uterine fibroids: results from a single center. Eur Radiol 5(25):1317-1328. https://doi. org/10.1186/2050-5736-3-S1-O99

17. Sainio T, Komar G, Saunavaara J, Suomi V, Joronen K, Perheentupa A, Viitala A, Sequeiros RB (2018) Wedged gel pad for bowel manipulation during MR-guided high-intensity focused ultrasound therapy to treat uterine fibroids: a case report. J Ther Ultrasound 6:1-10. https://doi.org/10.1186/s40349-018-0116-4

18. Park H, Yoon SW, Sokolov A (2015) Scaled signal intensity of uterine fibroids based on T2-weighted MR images: a potential objective method to determine the suitability for magnetic resonance-guided focused ultrasound surgery of uterine fibroids. Eur Radiol 25:3455-3458. https://doi.org/10.1007/s00330-015-3806-0

19. Kang SH, Lee SJ, Jeon GS, Yoon SW (2017) Scaled signal intensity of uterine fibroids on T2-weighted $\mathrm{mr}$ imaging as a predictor of the potential response to uterine fibroid embolization. J Vasc Interv Radiol 28:844-849. https://doi.org/10.1016/j. jvir.2017.02.003

20. Zavala Bojorquez JA, Jodoin PM, Bricq S, Walker PM, Brunotte F, Lalande A (2019) Automatic classification of tissues on pelvic MRI based on relaxation times and support vector machine. PLoS ONE 14:e0211944. https://doi.org/10.1371/journal.pone.0211944

21. Ikink ME, Voogt MJ, van den Bosch MA, Nijenhuis RJ, Keserci B, Kim YS, Vincken KL, Bartels LW (2014) Diffusion-weighted magnetic resonance imaging using different $b$-value combinations for the evaluation of treatment results after volumetric MR-guided high-intensity focused ultrasound ablation of uterine fibroids. Eur Radiol 24:2118-2127. https://doi.org/10.1007/s00330-014-3274-y

22. Spies JB, Bradley LD, Guido R, Maxwell GL, Levine BA, Coyne K (2010) Outcomes from leiomyoma therapies comparison with normal controls. Obstet Gynecol 116:641-652. https://doi. org/10.1097/AOG.0b013e3181ed36b3

23. Hectors SJ, Jacobs I, Heijman E, Keupp J, Berben M, Strijkers GJ, Grüll H, Nicolay K (2015) Multiparametric MRI analysis for the evaluation of MR-guided high intensity focused ultrasound tumor treatment. NMR Biomed 28:1125-1140. https://doi.org/10.1002/ nbm. 3350

24. Funaki K, Fukunishi H, Sawada K (2009) Clinical outcomes of magnetic resonance-guided focused ultrasound surgery for uterine myomas: 24-month follow-up. Ultrasound Obstet Gynecol 34:584-589. https://doi.org/10.1002/uog.7455

25. Yoon SW, Lee C, Cha SH, Yu JS, Na YJ, Kim KA, Jung SG, Kim SJ (2008) Patient selection guidelines in MR-guided focused ultrasound surgery of uterine fibroids: a pictorial guide to relevant findings in screening pelvic MRI. Eur Radiol 18:2997-3006. https ://doi.org/10.1007/s00330-008-1086-7

26. Sipola P, Ruuskanen A, Yawu L, Husso M, Vanninen R, Hippeläinen M, Manninen H (2010) Preinterventional quantitative magnetic resonance imaging predicts uterus and leiomyoma size reduction after uterine artery embolization. J Magn Reson Imaging 31:617-624. https://doi.org/10.1002/jmri.22063

27. Kilickesmez O, Bayramoglu S, Inci E, Cimilli T, Kayhan A (2009) Quantitative diffusion-weighted magnetic resonance imaging of normal and diseased uterine zones. Acta Radiol 50:340-347. https ://doi.org/10.1080/02841850902735858

28. Namimoto T, Awai K, Nakaura T, Yanaga Y, Hirai T, Yamashita Y (2009) Role of diffusion-weighted imaging in the diagnosis 
of gynecological diseases. Eur Radiol 19:745-760. https://doi. org/10.1007/s00330-008-1185-5

29. Andrews S, Yuan Q, Bailey A, Xi Y, Chopra R, Staruch R, Pedrosa I (2018) Multiparametric MRI characterization of funaki types of uterine fibroids considered for MR-guided high-intensity focused ultrasound (MR-HIFU) therapy. Acad Radiol 26:e9-e17. https:// doi.org/10.1016/j.acra.2018.05.012

30. Zhao W, Chen J, Zhang L, Li Q, Qin J, Peng S (2013) Feasibility of ultrasound-guided high intensity focused ultrasound ablating uterine fibroids with hyperintense on T2-weighted MR imaging. Eur J Radiol 82:e43-e49. https://doi.org/10.1016/j.ejrad 2012.08.020
31. Kim Y, Lim HK, Park MJ, Rhim H, Jung S, Sohn I, Kim T, Keserci B (2016) Screening magnetic resonance imaging-based prediction model for assessing immediate therapeutic response to magnetic resonance imaging-guided high-intensity focused ultrasound ablation of uterine fibroids. Invest Radiol 51:15-24. https ://doi.org/10.1097/RLI.0000000000000199

Publisher's Note Springer Nature remains neutral with regard to jurisdictional claims in published maps and institutional affiliations. 